\title{
Values of Chest Ultrasound and Blood Platelet in Diagnosis and Assessment the Severity of Community Acquired Pneumonia
}

\author{
Hussein Abd El-Fattah Mohammed ${ }^{1}$, Mohammed Ali Abboud ${ }^{2}$, \\ Fareed Shawky Basiony ${ }^{1}$, Ahmed Younes Younes Younes ${ }^{1 *}$
}

Departments of ${ }^{1}$ Chest Diseases and ${ }^{2}$ Radiodiagnosis, Faculty of Medicine, Al-Azhar University

*Corrosponding author: Ahmed Younes Younes Younes, E-mail: Shafehy100@ gmail.com

\begin{abstract}
Background: over the past 15 to 20 years, lung ultrasound has developed as an important modality for the bedside diagnosis of pneumonia both in the Emergency department and intensive care unit (ICU) settings, Platelets play a crucial role in antimicrobial host defenses and the coagulate system, it is hypothesized that an abnormal platelet count may be an important marker to assess severity of disease in patients with community acquired pneumonia (CAP). Aim of the Work: the aim of this study was to evaluate the role of chest ultrasound and blood platelet in diagnosis and assessment the severity of community acquired pneumonia. Patients and Methods: this was a prospective study carried out at Chest Department and Respiratory ICU at El-Hussein University hospital in the period from September 2017 to June 2018. The study included 50 cases of CAP; 29 cases male and 21 cases female with mean age $(48.32+15.62$ years $)$ admitted at the Chest Department and Respiratory ICU were enrolled in this study after obtaining informed consent. Results: the results of this study revealed that the transthoracic ultrasound can easily detect signs of consolidation and assess the success of pneumonia correlation with CURB 65 SCOR and blood platelet. Conclusion: thoracic ultrasound is a simple, bedside test used for diagnosis of pneumonia can differentiate between simple pneumonia and post stenotic pneumonia, atelectasis and mass. However, it is operator dependent and needs training.
\end{abstract}

Keywords: Thoracic Ultrasound, CURB 65 Scor, Blood Platelet.

\section{INTRODUCTION}

Community acquired pneumonia is the most common disease recorded worldwide; pathologically it is characterized by inflammatory process that involve alveolar space or interstitial tissue or both ${ }^{(\mathbf{1})}$.

The diagnosis of pneumonia is based on clinical presentation, laboratory tests, and chest imaging. The utility of chest radiography is limited compared with chest CT that considers the gold stander for diagnosis of pneumonia ${ }^{(\mathbf{1})}$.

Chest ultrasound has been shown to be highly effective in evaluation arrange of pathogenic pulmonary study and its one of the most widely application in evaluation of pneumonia ${ }^{(2)}$.

Platelets play a crucial role in antimicrobial host defenses and the coagulate system; it is hypothesized that an abnormal platelet count may be an important marker to assess severity of disease in patients with $\mathrm{CAP}^{(3)}$.

\section{AIM OF THE WORK}

The aim of this study is to evaluate the role of chest ultrasound and blood platelet in diagnosis and assessment the severity of community acquired pneumonia.

\section{PATIENTS AND METHODS}

This was a prospective study carried out at Chest Department and Respiratory ICU at El-Hussein University hospital in the period from September 2017 to June 2018. The study included 50 cases of CAP; 29 cases male and 21 cases female with mean age $(48.32+15.62$ years) admitted at the Chest Department and Respiratory ICU were enrolled in this study after obtaining informed consent. The study was approved by the Ethics Board of AlAzhar University.

All cases were subjected to the following: Full history taking, Full clinical examination, Vital signs (pulse, blood pressure, temperature, respiratory rate, Systemic examination to exclude co existing systemic disease, Chest examination for signs of consolidation e.g. rales and bronchial breathing, Routine laboratory investigations, Complete blood picture was done on the first day of admission and on 7th day to assess the response of platelet count to inflammatory process, Hepatic and renal profiles, Blood coagulation profiles, Serum electrolyte $(\mathrm{Na}-\mathrm{K}$ - 
Mg-Ca), Erythrocyte sedimentation rate (ESR). Radiological evaluation such as Plain chest X-ray: A postero-anterior and lateral views were performed on the first day of admission and on 7th day, Chest ultrasound: It was performed within 24 hours after plain chest $\mathrm{x}$ - ray to assess: Location, shape and size of consolidation, Presence of necrotic areas in consolidation, Presence of air bronchograms, Presence of complicated pleural effusion, Thoracic sonography was done using (Sonoscape A8 Medical Systems, Shenzhen, China), Probes: The convex probe was used for visualization of normal structures and artifacts, consolidations, the high-frequency linear array probe for detailed examination of the pleura while phased array cardiac probe for visualization of small lesions and for rapid cardiac assessment e.g. for pericardial effusion,Preparation: No specific patient preparation was done,Theater: Thoracic ultrasound was performed at the ultrasonography unit in chest department at ElHussein University hospital,Position: supine for front and sitting for back during inspiration and expiration, if necessary in combination with respiratory maneuvers such as coughing or "sniffing." Raising the arms and crossing them behind the head causes intercostals spaces to be extended and facilitates access,Settings: Optimal B-mode and M-mode settings of gain, depth, focal zone placement, and compression were individually adjusted for each patient according to thoracic ultrasound protocol.

Exclusion criteria: Patients with previous use of oral corticosteroids, Other immunosuppressive therapy, tuberculosis, HIV infection, active solid or hematologic neoplasm, Hematologic disease involving platelets and/or leukocytes, such as essential thrombocytosis or myelodysplastic syndrome, Patients on anti-platelet or anticoagulant drugs, Hospitalization within the preceding 21 days.

Statistics: Statistical analysis of the present study was carried out using the SPSS computer package version 18.0 (SPSS Inc., Chicago, IL, USA) and used the mean, standard error, student t- test, Chi-square, The mean \pm SD. Unpaired Student t-test was used to compare between two groups in quantitative data, while number and \% were used for qualitative variables. Chi-square the hypothesis that the row and column variables are independent, without indicating strength or direction of the relationship, Pearson chisquare and likelihood-ratio chi-square. Fisher's exact test and Yates' corrected chi-square are computed for $2 \times 2$ tables. Chi-square the hypothesis that the row and column variables are independent, without indicating strength or direction of the relationship,Pearson chisquare and likelihood-ratio chi-square, In order to assess the differences in frequency of qualitative variables, Chi-square (X2) test or Fisher's exact test (FET) was applied when appropriate,In order to assess differences in means of quantitative variables, Independent samples t-test or One-Way ANOVA test was applied when appropriate, while MannWhitney $U$ test was used for non-parametric statistics,Data are expressed as median range, $\mathrm{P}$ value assessed via K.W.=Kruskal Walis test for group comparison, Statistical significance was defined as $\mathrm{P} \leq 0.05$. A , B ,C,D means significant difference between corresponding groups by Mann-Whitney multiple comparison,According to $(\mathrm{MC}=$ Monte Carlo test, $\mathrm{P}$ value is significant if $\leq 0.05$. )

Ethical consideration: Ethical clearance was obtained from the Research Ethical Committee at Al-Hussein University Hospital. A signed written informed consent from patients or their relatives was obtained. Privacy and confidentiality were maintained throughout the study process. Subjects or their relatives received written notification of the intervention results.

\section{RESULTS}

Table (1): Demographic data of the studied group

\begin{tabular}{|l|c|c|}
\hline \multicolumn{1}{|c|}{ Variables } & No & \% \\
\hline Age & & \\
Mean $\pm(\mathrm{SD})$ & $48.32 \pm 15.62$ & \\
Min-max & $19-84$ & \\
\hline Sex & 29 & 58 \\
Male & 21 & 42 \\
Female & 22 & \\
\hline Smoking status & 28 & 44 \\
Non smoker & & \\
Smoker & 36 & 72 \\
\hline Residence & 14 & 28 \\
Urban & & \\
Rural &
\end{tabular}

This table shows that the mean age of the studied cases ranged from 19 to 84 years with a mean of $48.32 \pm 15.62$. Also show that male represent $58 \%$ while female represent $42 \%$. 
The most studied individuals were smokers representing $56 \%$. Moreover, the table shows that urban patients represent $72 \%$ while rural patients represent $28 \%$.

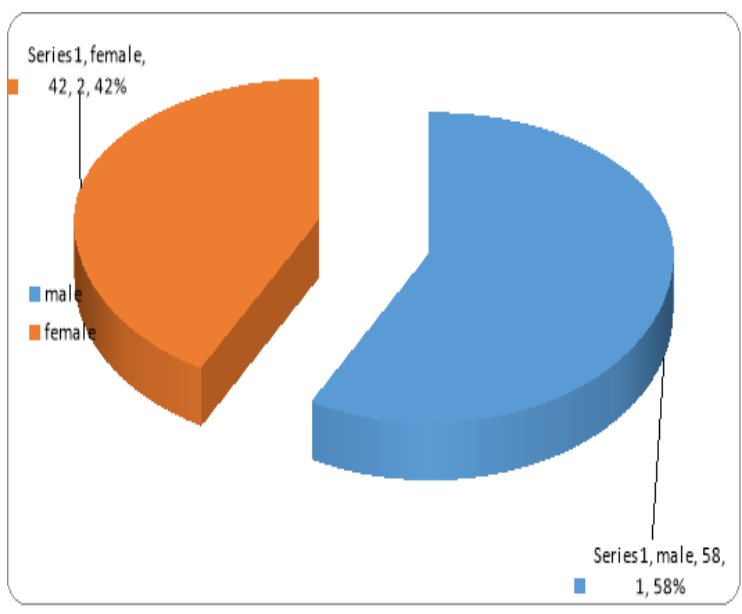

Figure (1): Sex distribution of the studied groups.

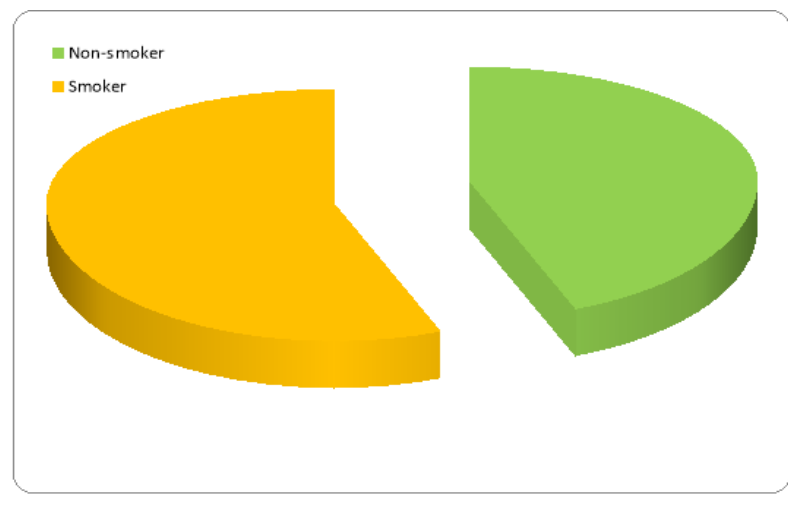

Figure (2): Smoking status of the studied group.

Table (2): Ultrasound final diagnosis of studied group

\begin{tabular}{|l|c|c|}
\hline \multicolumn{1}{|c|}{ Variables } & No. & $\%$ \\
\hline Pneumonic consolidation only & 30 & 60 \\
\hline $\begin{array}{l}\text { Pneumonic consolidation with cavity } \\
\text { formation }\end{array}$ & 3 & 6 \\
\hline Pneumonic consolidation with collapse & 12 & 24 \\
\hline $\begin{array}{l}\text { Pneumonic consolidation with pleural } \\
\text { effusion }\end{array}$ & 5 & 10 \\
\hline Total & 50 & 100 \\
\hline
\end{tabular}

This table showed that 30 cases diagnosed as pneumonia only, 3 cases were pneumonia with cavity formation, 12 cases had atelectasis, and 5 cases had Pneumonia with pleural effusion.

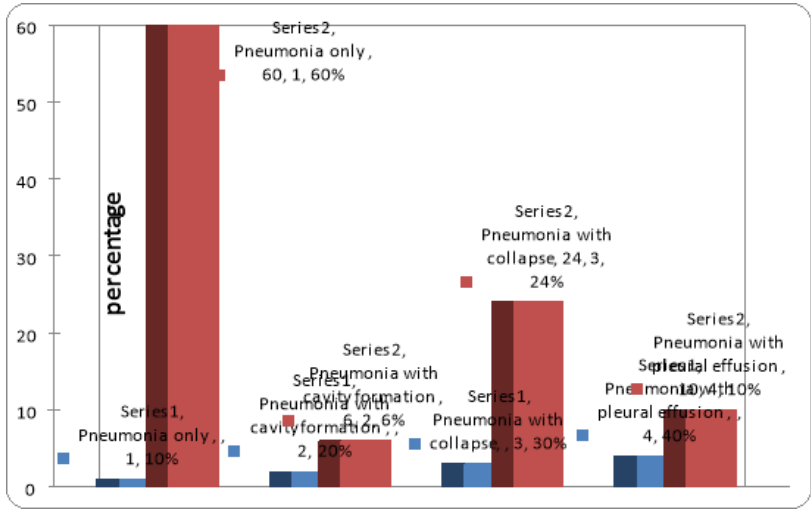

Figure (3): Final diagnosis of cases by chest US.

Table (3): CURB-65 score in studied group:

\begin{tabular}{|l|c|c|}
\hline \multicolumn{1}{|c|}{ Variables } & No. & \% \\
\hline Grade 0 & 20 & 40 \\
\hline Grade 1 & 10 & 20 \\
\hline Grade 2 & 7 & 14 \\
\hline Grade $\geq 3$ & 13 & 26 \\
\hline
\end{tabular}

This table show that the distribution of cases according to CURB 65 score for detection of pneumonia severity; 20 cases included in grade 0,10 cases included in grade 1,7 cases were included in grade 3 and 13 cases included in grade more than 3 .

Table (4): Platelet count in studied group

\begin{tabular}{|l|c|c|}
\hline \multicolumn{1}{|c|}{ Variables } & No. & \% \\
\hline Normal platelet count & 13 & 26 \\
\hline Thrombocytosis & 8 & 16 \\
\hline Thrombocytopenia & 29 & 58 \\
\hline Total & 50 & 100 \\
\hline
\end{tabular}

This table showed that 29 patients (58 $\%$ had thrombocytopenia (platelet count $<150,000), 13$ patients $(26 \%)$ had normal platelet count $(150,000-400,000)$ and only 8 patients $(16 \%)$ had thrombocytosis (platelet count $>400,000$ ).

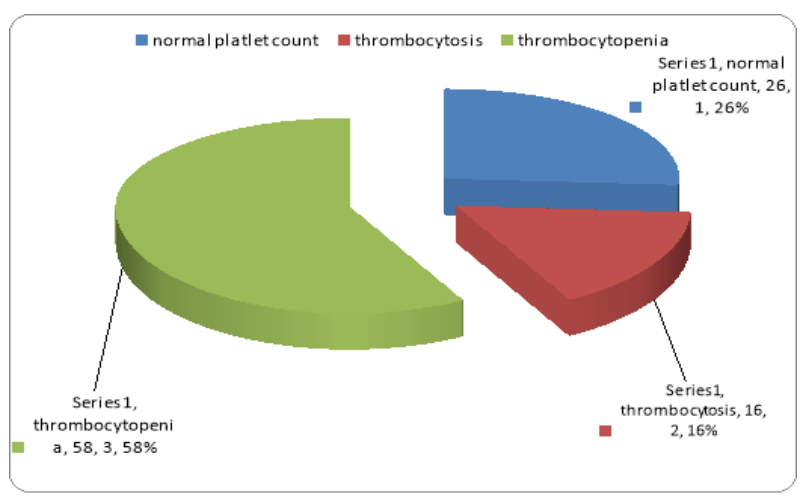

Figure (4): Platelet count in studied group. 
Table (5): Relationship between platelet count and CURB 65 score:

\begin{tabular}{|c|c|c|c|c|}
\hline \multirow{3}{*}{ Variables } & \multicolumn{3}{|c|}{ Types of platelet count } & \multirow[b]{2}{*}{$P$ value } \\
\hline & Normal & Low & High & \\
\hline & \multicolumn{3}{|c|}{ Median (min-max) } & \\
\hline CURB 65 score & $\begin{array}{c}0^{A} \\
(0-1)\end{array}$ & $\begin{array}{c}2^{\mathrm{AB}} \\
(0-4)\end{array}$ & $\begin{array}{c}0^{\mathrm{B}} \\
(0-1)\end{array}$ & $<0.001$ \\
\hline
\end{tabular}

This table shows that that patient with thrombocytopenia had significant higher CURB 65 score as a score for severity of CAP.

Table (6): Relation between extent of consolidation and CURB 65 score

\begin{tabular}{|c|c|c|}
\hline & $\begin{array}{c}\text { Extent } \\
\text { Mean } \pm \text { SD }\end{array}$ & P value \\
\hline CURB score & & \\
$\mathbf{0}$ & $4.2 \pm 1.3$ & \\
$\mathbf{1}$ & $4.6 \pm 1.4$ & $\leq 0.001$ \\
$\mathbf{2}$ & $6.8 \pm 1.1$ & \\
$\mathbf{3}$ & $6.9 \pm 0.8$ & \\
$\mathbf{4}$ & $7.2 \pm 0.9$ & \\
\hline
\end{tabular}

Data are presented as mean \pm standard deviation. $P$ value assessed by one way ANNOVA. $\mathrm{p}$ value less than 0.05 is considered statistically significant

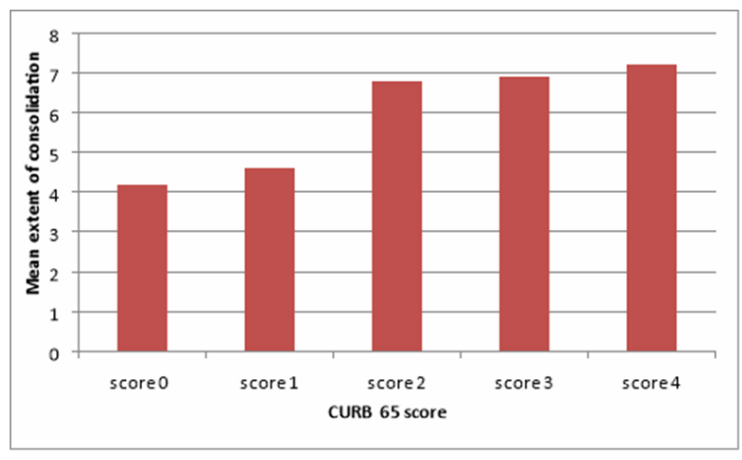

Figure (5): Extent of consolidation according to CURB 65 score.

\section{DISCUSSION}

Despite the development of new diagnostic tests and new antimicrobial agents; community-acquired pneumonia (CAP) still has high morbidity and mortality worldwide and is associated with moderate health cost.

In this study, pneumonia was clinically suspected in 50 cases admitted at the Chest Department and Respiratory ICU at AlAzhar University Hospital after obtaining informed consent and exclusion of patients who did not match with criteria of this study. This study was a prospective one done to compare between platelet count abnormality and thoracic US with CURB-65 score in determination of the severity of communityacquired pneumonia cases.

As regard age of the studied cases; it ranged from 19 to 84 years with a mean of $48.32 \pm 15.62($ table 1$)$.

The study agree with that done by ElMaraghy et al. ${ }^{(4)}$. The results showed that the age ranged from 17 to 84 years with a mean of $60 \pm 17$ years.

Pneumonia can occur at any age, but its incidence increases significantly with advanced age ${ }^{(5)}$.

Also the study agree with that done by Jain et al., $2015^{(6)}$ found an increased incidence of pneumonia with increasing age, with the highest rates among adults aged.

As regard gender, residence, smoking in our study showed that male represent $58 \%$ and female represent $42 \%$. This may be explained by high prevalence of smoking in males which is known to increase risk of respiratory infections and most studied individuals were smokers representing $56 \%$. Moreover, the urban patients represent $72 \%$ and rural; represent $28 \%$; Table (1), figure (1, 2).

As regard to smoking, there were 28 smokers (all were males) and 22 non-smokers. Smoking is associated with colonization by pathogenic bacteria and an increased risk of lung infections ${ }^{(7)}$.

As regards to diagnosis of cases by US, the result showed that 30 cases were pneumonia only, 3 cases were pneumonia with cavity formation, 12 cases had Pneumonia with lung collapse and 5 cases had pneumonia with pleural effusion; Table (2), figure (3).

The lung is not an ideal target for ultrasonography, but once fluid or solid material has accumulated in the lung it can more easily be visualized on an ultrasound scan. That is the reason that lung ultrasonography is being increasingly utilized in the diagnosis of pneumonia ${ }^{(8)}$.

As regards the severity of pneumonia by using CURB-65 score; the result showed that 20 cases included in grade 0,10 cases included in grade 1,7 cases were included in grade 3 and 13 cases included in grade more than 3 (table 3 ). 
As regards to platelet count in studied patients, the results showed that 29 patient $(58 \%)$ had thrombocytopenia (platelet count <150,000), 13 patients $(26 \%)$ had normal platelet count $(150,000-400,000)$ and only 8 patients $(16 \%)$ had thrombocytosis (platelet count $>400,000$ ) (table 4).

In the study done by Lamiaa and Yousef ${ }^{\left({ }^{(9)}\right.}$ normal platelet count was detected in $64(67.4 \%)$ patients, thrombocytosis in 26 $(27.4 \%)$ patients and thrombocytopenia in 5 $(5.2 \%)$ patients.

In study done by El-Maraghy et al. ${ }^{(4)}$ show that there was no shows that there was a significant statistical relation between the occurrence of respiratory complications and both thrombocytopenia and thrombocytosis by using chi-square test.

In contrast Prina et al. ${ }^{(10)}$ evaluated 3010 patients with CAP, where 2423 met their inclusion criteria and found that thrombocytosis presented in 204 (8\%) and thrombocytopenia presented in 53 (2\%), patients with thrombocytosis had lower CURB-65 score at admission however patients with thrombocytosis and those with thrombocytopenia had higher mortality rate in accordance with our results.

\section{CONCLUSION}

Thoracic ultrasound is a simple, bedside test used for diagnosis of pneumonia and can differentiate between simple pneumonia and post stenotic pneumonia, atelectasis and mass. However, it is operator dependent and needs training, Thoracic ultrasound should be included in initial assessment of patients with suspected pneumonia, follow-up and assessment severity of pneumonia. The abnormal platelet count is associated with severity of disease and more frequently complications in patients with CAP.

\section{REFERRENCES}

1. File TM and Marrie TJ (2010): Burden of community-acquired pneumonia in North American adults. Post grad Med., 122:130-141.
2. Welte T, Torres A, Nathwani D (2012): Clinical and economic burden of community-acquired pneumonia among adults in Europe. Thorax, 67:71-9.

3. Mandell LA, Wunderink RG, Anzueto A (2007): Infectious Diseases Society of America; American Thoracic Society. Consensus guidelines on the management of community-acquired pneumonia in adults. Clinical infectious diseases, 44(2): S27-S72.

4. El Maraghy AA, Abdel Fattah EB, Ahmed MS (2015): Platelet count: Is it a possible marker for severity and outcome of community-acquired pneumonia? Egyptian Journal of Chest Diseases and Tuberculosis, 65: 499-504.

5. Cillóniz $C$ and Torres A (2016): Community-Acquired Pneumonia 20002015: What is New?. Barcelona Respiratory Network Reviews; 2:253-73.

6. Jain S, Williams DJ, Arnold SR (2015): Community-acquired pneumonia requiring hospitalization among US children. New England Journal of Medicine, 372(9): 835845.

7. Kohl hammer Y, Schwartz M, Raspe H (2005): Risk factors for community acquired pneumonia (CAP). A systematic review. Deutsche medizinische Wochenschrift , 130(8): 381-386.

8. Roić G (2016): Lung ultrasound in the diagnosis of pediatric pneumonia: are we ready for routine use? Act aMedica Academica, 45(1): 82-85.

9. Lamiaa H. Shaaban, Yousef Ahmed (2015): Hemogram values in community acquired pneumonia. Egyptian Journal of Chest Diseases and Tuberculosis, 64: 617623.

10. Prina E, Ferrer M, Ranzani OT, Polverino E, Cillo' niz C, Moreno E, Mensa J, Montull B, Mene' ndez R, Cosentini R, Torres A (2013): Thrombocytosis a marker of poor outcome in community acquired pneumonia, Chest, 143(3): 767-775. 